\title{
Recent results from the NA62 experiment at CERN
}

\author{
Riccardo Aliberti* ${ }^{\dagger}$ \\ Institut für Physik, Johannes Gutenberg-Universität, Mainz \\ E-mail: riccardo.aliberti@cern.ch
}

NA62 is a fixed target experiment located at the CERN SPS which aims to perform flavour physics studies in the charged kaon sector with an unprecedented precision. Due to the abundance of kaon decays with a $\pi^{o}$ in the final state, NA62 is also a perfect environment to measure the neutral pion characteristics. NA62 collected a large sample of charged kaon decays with a highly efficient trigger for decays into electrons in 2007. We report about the measurement of the $\pi^{o}$ electromagnetic transition form factor slope parameter in the time-like region.

Between 2008 and 2014 the NA62 apparatus underwent an intense upgrade phase to improve the physics sensitivity of the experiment to precisely measure the $\mathrm{Br}\left(K^{+} \rightarrow \pi^{+} v \bar{v}\right)$. NA62 took data in pilot runs in 2014 and 2015 reaching the final designed beam intensity. The quality of data acquired in view of the final measurement is presented.

XXIV International Workshop on Deep-Inelastic Scattering and Related Subjects

11-15 April, 2016

DESY Hamburg, Germany

\footnotetext{
* Speaker.

${ }^{\dagger}$ for the NA62 Collaboration: G. Aglieri Rinella, R. Aliberti, F. Ambrosino, R. Ammendola, B. Angelucci, A. Antonelli, G. Anzivino, R. Arcidiacono, I. Azhinenko, S. Balev, M. Barbanera, J. Bendotti, A. Biagioni, L. Bician, C. Biino, A. Bizzeti, T. Blazek, A. Blik, B. Bloch-Devaux, V. Bolotov, V. Bonaiuto, M. Boretto, M. Bragadireanu, D. Britton, G. Britvich, M.B. Brunetti, D. Bryman, F. Bucci, F. Butin, E. Capitolo, C. Capoccia, T. Capussela, A. Cassese, A. Catinaccio, A. Cecchetti, A. Ceccucci, P. Cenci, V. Cerny, C. Cerri, B. Checcucci, O. Chikilev, S. Chiozzi, R. Ciaranfi, G. Collazuol, A. Conovaloff, P. Cooke, P. Cooper, G. Corradi, E. Cortina Gil, F. Costantini, F. Cotorobai, A. Cotta Ramusino, D. Coward, G. D’Agostini, J. Dainton, P. Dalpiaz, H. Danielsson, J. Degrange, N. De Simone, D. Di Filippo, L. Di Lella, S. Di Lorenzo, N. Dixon, N. Doble, B. Dobrich, V. Duk, V. Elsha, J. Engelfried, T. Enik, N. Estrada, V. Falaleev, R. Fantechi, V. Fascianelli, L. Federici, S. Fedotov, M. Fiorini, J. Fry, J. Fu, A. Fucci, L. Fulton, S. Gallorini, S. Galeotti, E. Gamberini, L. Gatignon, G. Georgiev, A. Gianoli, M. Giorgi, S. Giudici, L. Glonti, A. Goncalves Martins, F. Gonnella, E. Goudzovski, R. Guida, E. Gushchin, F. Hahn, B. Hallgren, H. Heath, F. Herman, T. Husek, O. Hutanu, D. Hutchcroft, L. Iacobuzio, E. Iacopini, E. Imbergamo, O. Jamet, P. Jarron, E. Jones, T. Jones K. Kampf, J. Kaplon, V. Kekelidze, S. Kholodenko, G. Khoriauli, A. Khotyantsev, A. Khudyakov, Yu. Kiryushin, A. Kleimenova, K. Kleinknecht, A. Kluge, M. Koval, V. Kozhuharov, M. Krivda, Z. Kucerova, Yu. Kudenko, J. Kunze, G. Lamanna, G. Latino, C. Lazzeroni, G. Lehmann-Miotto, R. Lenci, M. Lenti, E. Leonardi, P. Lichard, R. Lietava, L. Litov, R. Lollini, D. Lomidze, A. Lonardo, M. Lupi, N. Lurkin, K. McCormick, D. Madigozhin, G. Maire, C. Mandeiro, I. Mannelli, G. Mannocchi, A. Mapelli, F. Marchetto, R. Marchevski, S. Martellotti, P. Massarotti, K. Massri, P. Matak, E. Maurice, A. Mefodev, E. Menichetti, E. Minucci, M. Mirra, M. Misheva, N. Molokanova, J. Morant, M. Morel, M. Moulson, S. Movchan, D. Munday, M. Napolitano, I. Neri, F. Newson, A. Norton, M. Noy, G. Nuessle, T. Numao, V. Obraztsov, A. Ostankov, S. Padolski, R. Page, V. Palladino, G. Paoluzzi, C. Parkinson, E. Pedreschi, M. Pepe, F. Perez Gomez, M. Perrin-Terrin, L. Peruzzo, P. Petrov, F. Petrucci, R. Piandani, M. Piccini, D. Pietreanu, J. Pinzino, I. Polenkevich, L. Pontisso, Yu. Potrebenikov, D. Protopopescu, F. Raffaelli, M. Raggi, P. Riedler, A. Romano, P. Rubin, G. Ruggiero, V. Russo, V. Ryjov, A. Salamon, G. Salina, V. Samsonov, C. Santoni, G. Saracino, F. Sargeni, V. Semenov, A. Sergi, M. Serra, A. Shaikhiev, S. Shkarovskiy, I. Skillicorn, D. Soldi, A. Sotnikov, V. Sugonyaev, M. Sozzi, T. Spadaro, F. Spinella, R. Staley, A. Sturgess, P. Sutcliffe, N. Szilasi, D. Tagnani, S. Trilov, M. Valdata-Nappi, P. Valente, M. Vasile, T. Vassilieva, B. Velghe, M. Veltri, S. Venditti, P. Vicini, R. Volpe, M. Vormstein, H. Wahl, R. Wanke, P. Wertelaers, A. Winhart, R. Winston, B. Wrona, O. Yushchenko, M. Zamkovsky, A. Zinchenko.
} 


\section{The NA62 Experiment}

NA62 is a fixed target experiment located at the North Area of the European Center for Nuclear Research (CERN). The proton beam from the SPS accelerator is used to produce a secondary hadron beam and to perform flavor physics studies mainly in the charged kaon sector. NA62 took over the NA48 ${ }^{[1]}$ experiment physics program and in 2007 acquired for the first time data using the same beam line and apparatus as in NA48. The purpose of the run was to study the lepton universality through the measurement of the $R_{K}=\frac{K^{ \pm} \rightarrow e^{ \pm} v}{K^{ \pm} \rightarrow \mu^{ \pm} v}$ parameter ${ }^{[2]}$. Starting from 2008 the experiment underwent an intense phase of $R \& D$ finalized to the upgrade of the apparatus to push further the physics sensitivity. The new detector was optimized for the precise measurement of the $K^{+} \rightarrow \pi^{+} v \bar{v}$ branching ratio and has taken data for the first time in 2014/2015.

\section{Measurement of the $\pi^{o}$ Transition Form Factor}

The neutral pion is the lightest meson and plays a very important role in the study of lowenergy properties of strong nuclear force. The main decay mode of the $\pi^{o}$ has two photons in final state, $B r_{\left(\pi^{o} \rightarrow \gamma \gamma\right)}=(98.823 \pm 0.034) \%[3]$, and is followed by the so called Dalitz decay, $B r_{\left(\pi_{D}^{o} \rightarrow e^{+} e^{-} \gamma\right)}=(1.174 \pm 0.035) \%[3]$. The differential decay amplitude of the $\pi_{D}^{o}$ normalized to $\pi_{\gamma \gamma}^{0}$ reads

$$
\frac{1}{\Gamma\left(\pi_{\gamma \gamma}^{o}\right)} \frac{d^{2} \Gamma\left(\pi_{D}^{o}\right)}{d x d y}=\frac{\alpha}{4 \pi} \frac{(1-x)^{3}}{x}\left(1+y^{2}+\frac{r^{2}}{x}\right)|F(x)|^{2}(1+\delta(x, y))
$$

where $x=\frac{\left(p_{e^{+}}+p_{e^{-}}\right)^{2}}{m_{\pi^{o}}^{2}}, y=2 p_{\pi^{o}} \frac{\left(p_{e^{+}}-p_{e^{-}}\right)}{m_{\pi^{o}}^{2}(1-x)}, F(x)$ corresponds to the semi off-shell transition form factor (TFF) describing the hadronic physics in the $\left(\gamma^{*} \rightarrow e^{+} e^{-}\right)$vertex and $\delta(x, y)$ contains the radiative corrections to the $\pi_{D}^{o}$ decay. The TFF is usually defined as $F(x)=1+a x$ where $a$ is the Transition Form Factor slope. The $\pi^{o}$ TFF contribute to the calculation of several observable quantities like the rate of $\pi^{o} \rightarrow e^{+} e^{-}$and the anomalous magnetic momentum of the muon $(g-2)_{\mu}$. The TFF has been object of extensive theoretical studies ${ }^{[4][5]}$ and its slope experimentally measured in both time-like and space-like domain.

Many of the main decay modes of charge kaons have a $\pi^{o}$ in the final state. The high intensity kaon beam in NA62 makes it the perfect environment to study $\pi^{\circ}$ physics. During the 2007 data taking $2 \times 10^{10} K^{ \pm}$decays were collected, allowing to measure the $\pi^{o}$ TFF slope with a precision never reached before. The parameter $a$ is extracted through the study of the slope in the $x$ spectrum following the integration of equation (2.1) over the $y$ variable. The radiative corrections have been carefully studied and the latest calculation ${ }^{[6]}$ has been implemented in the Monte Carlo simulation of the $\pi_{D}^{o}$ decay.

\subsection{The NA62 apparatus in 2007}

The NA48 beam line and apparatus were used in the NA62 2007 run, but with different beam parameters. The primary $400 \mathrm{GeV} / c$ proton beam from the SPS impinged on a beryllium target producing a secondary hadron beam. The charged particles in the beam were then transported, focused and filtered in momentum in the $100 \mathrm{~m}$ long beam line which was kept in vacuum. Downstream the vacuum region, the apparatus provided an efficient detection of the kaon decay products. 
A magnetic spectrometer with drift chambers in Helium traced the charged particles, a Hodoscope (CHOD), made of two perpendicular planes of scintillators, provided the trigger and the timing for charged particles with sub-nanosecond resolution. The energy from electrons and photons was measured by a quasi-homogeneous electromagnetic calorimeter filled with Liquid Krypton (LKr). The trigger in 2007 was optimized for electron events by measuring the energy deposit in LKr.

\subsection{Event selection and preliminary results}

A selection of $K^{ \pm} \rightarrow \pi^{ \pm} \pi^{o}$ events with $\pi^{o} \rightarrow e^{+} e^{-} \gamma$ is performed with a full kinematic reconstruction. A clean three-track event topology is required, with all tracks coming from the same vertex that has to be located inside the fiducial decay region. A single LKr cluster, separated in space from the track impact points at the calorimeter surface, identifies the photon. The total reconstructed 4-momentum from photon and tracks has to be consistent with the nominal beam energy and direction within the resolution. Assuming the track with charge opposite to the $K^{ \pm}$to be the $e^{\mp}$, the event is selected if the other two tracks satisfy only one of the the two possible mass hypotheses $\left(e^{ \pm}, \pi^{ \pm}\right)$for the $K^{ \pm} \rightarrow \pi^{ \pm} \pi_{D}^{o}$ decay kinematics, within the reconstructed resolution of the $\pi^{o}$ and kaon mass. Finally, in order to keep the consistency between the MC simulation and the online trigger configuration in the data, the total energy deposit inside the $\mathrm{LKr}$ has to be greater than $14 \mathrm{GeV}$ and one of the $e^{+} / e^{-}$tracks has to have momentum greater than $5.5 \mathrm{GeV} / c$ and must deposit more than $80 \%$ of its energy in the calorimeter. Only events with $x>0.01$ are selected since the acceptance for events with $x \leq 0.01$ is not well described in the MC simulation.
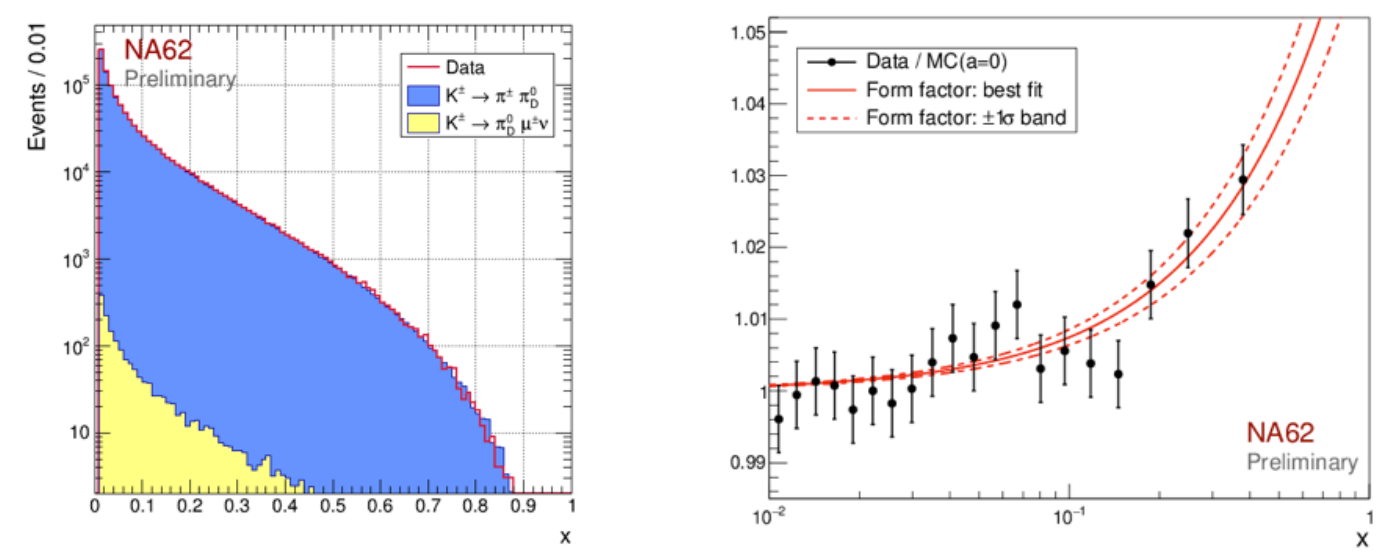

Figure 1: On the left the $x$ spectrum in data and MC. On the right the TFF slope parameter fit

About $1.05 \times 10^{6} \pi_{D}^{o}$ decays were fully reconstructed. The $\pi^{o}$ TFF slope parameter is then determined by fitting the ratio between the $x$ spectrum from data and from MC simulation (Fig.1). The preliminary measurement of the TFF slope parameter is $a=\left(3.70 \pm 0.53_{\text {stat }} \pm 0.35_{\text {syst }}\right) \times 10^{-2}$ with a $\chi^{2} /$ n.d.f. $=52.5 / 49$. The main contributions to the systematic uncertainty are given by the momentum spectrum simulation and by the spectrometer momentum scale calibration. The result improves the experimental measurement of the slope parameter in the time-like region by more than one order of magnitude and is compatible with the theoretical predictions (within the uncertainties). 


\section{The search for $K^{+} \rightarrow \pi^{+} v \bar{v}$ at $\mathbf{N A 6 2}$}

The rare decay $K^{+} \rightarrow \pi^{+} v \bar{v}$ is a Flavor Changing Neutral Current (FCNC) process dominated by the short distance interactions and therefore highly suppressed. The clean theoretical environment allows to have a precise computation of the Standard Model prediction: $B r_{S M}\left(K^{+} \rightarrow \pi^{+} v \bar{v}\right)=$ $(9.11 \pm 0.72) \times 10^{-11[7]}$ where the main sources of uncertainty are the CKM matrix parameters.

Many theoretical models of physics beyond the SM predict a deviation from the $K^{+} \rightarrow \pi^{+} v \bar{v}$ SM branching ratio value. The high sensitivity of this process to the existence of new leptons or heavy mesons up to the $100 \mathrm{TeV}$ scale makes it a golden channel in the search of physics beyond the SM.

The data from the E787 and E949 experiments at Brookhaven National Laboratories (BNL) combined provide the first experimental measurement of $B r_{\exp }\left(K^{+} \rightarrow \pi^{+} v \bar{v}\right)=\left(17.3_{-10.5}^{+11.5}\right) \times$

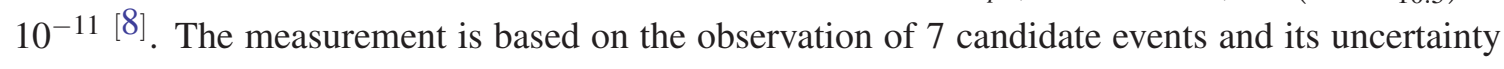
does not allow to probe precisely the standard model validity.

\subsection{Measurement strategy and NA62 apparatus}

The aim of the NA62 experiment is to collect around $100 K^{+} \rightarrow \pi^{+} v \bar{v}$ signal events in order to measure the branching ratio with $10 \%$ accuracy before the end of $2018^{[9]}$. The experiment plans to collect $10^{13}$ kaon decays in the foreseen data taking period by using the $400 \mathrm{GeV} / \mathrm{c}$ proton beam from the CERN SPS accelerator. Assuming a 10\% acceptance the background signal ratio should be kept below $20 \%$ to achieve the design sensitivity, which implies a background suppression at the level of $10^{12}[10]$. The tiny branching ratio combined with the weak signature of the signal requires a very challenging rejection of the main $K^{+}$decay modes. The key to achieve the claimed sensitivity are: the rejection of beam induced background, a precise measurement of kinematics, an efficient particle identification and a hermetic photon detection.

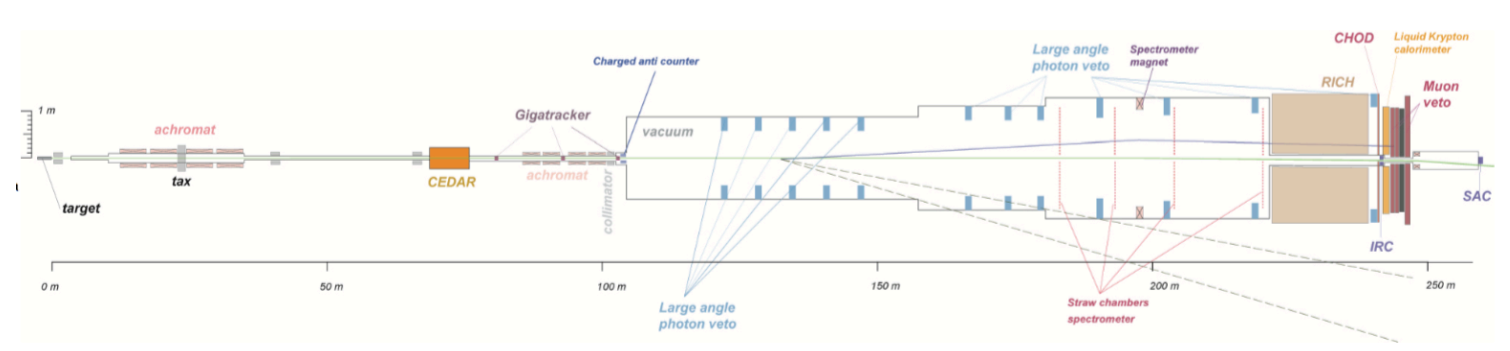

Beam induced background The $400 \mathrm{GeV}$ protons beam from SPS impinge on a Beryllium target resulting in a secondary hadron beam which reaches the experiment with a central momentum of $(75 \pm 0.8) \mathrm{GeV} / c$. The kaons represent $6 \%$ of the total $750 \mathrm{MHz}$ particle rate. The remaining components are protons (10\%) and pions (74\%). A Cherenkov detector filled with Nitrogen (Cedar) provides the kaon identification with 100 ps resolution. The precise timing from the Cedar allows to reject $\pi^{+} \rightarrow \mu^{+} v$ that could mimic the signal. A series of scintillator arrays (CHANTI) around the beam line are used to veto the interaction of particles inside the beam spectrometer. 
Measurement of Kinematics The suppression of background from the reconstruction of kinematic variables is required to be at the level of $10^{-5}$ on the main kaon decays as shown in figure 2. Two regions in the squared missing mass spectrum $\left(M_{\text {miss }}^{2}=\left(P_{K^{+}}-P_{\pi^{+}}\right)^{2}\right)$ have been defined on the two sides of $K^{+} \rightarrow \pi^{+} \pi^{o}$ peak. A precise measurement of both $K^{+}$and $\pi^{+}$direction and momentum with a sub-percent resolution is performed by two spectrometer systems. The beam spectrometer Gigatracker (GTK), placed right after the Cedar detector, is composed by three, $300 \mu m$ thick,

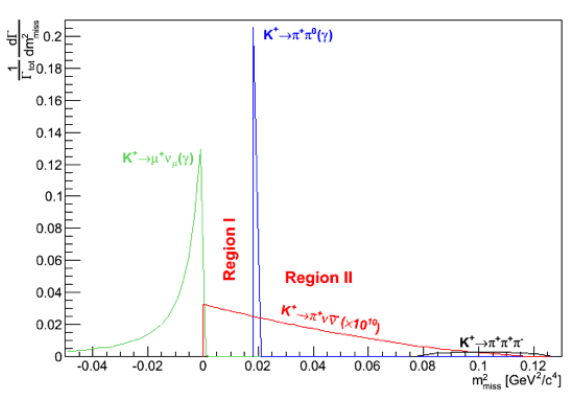

Figure 2: Distribution of squared missing mass for the main kaon decays silicon pixel stations. The GTK faces the full beam rate and for this reason the timing performances are crucial, the single hit time resolution is at the level of $200 \mathrm{ps.} \mathrm{A} \mathrm{magnetic} \mathrm{spectrometer}$ is used to track the kaon decay products. It is composed of 4 stations of straw tube chambers resulting in a relative momentum resolution $\left(\sigma_{p} / p\right)$ better than $0.3 \%$. The combined data from the GTK and STRAW detectors allow a reconstructed vertex resolution at the mm level. The last station of the GTK define the start of the fiducial decay region, which ends at the first station of the STRAW tube spectrometer.

Particle Identification The kaon decay $K^{+} \rightarrow \mu^{+} v$ with a branching ratio of $63.6 \%$ [3] represents the main background source for the $K^{+} \rightarrow \pi^{+} v \bar{v}$ measurement. In order to keep this background under control a muon suppression factor of $10^{8}$ is required. The NA62 detector has been designed in order to provide a very efficient Particle Identification (PID). The system includes a Ring Cherenkov detector (RICH), $17 \mathrm{~m}$ long, filled with Neon and a system of calorimeters which allows to disentangle $\pi / \mu / e$ by studying the energy share between the electromagnetic calorimeter ( $\mathrm{LKr}$ ) and the hadron calorimeter (HAC). Following a $80 \mathrm{~cm}$ thick iron wall a fast muon veto (MUV3) provides an online veto trigger with a $300 \mathrm{ps}$ time resolution.

Photon Veto A hermetic coverage of the experiment against photons is fundamental to veto all the kaon decays containing a $\pi^{o}$ in the final state $(B r \sim 30 \%[3])$ with an inefficiency at the level of $10^{-8}$. The NA62 experiment is equipped with three different type of detectors devoted to the photon detection from 0 up to 50 mrad: 12 stations of Large Angle Veto (LAV), an electromagnetic calorimeter (LKr) and two Small Angle Vetoes (IRC and SAC) for photons down to 0 mrad.

\subsection{Data Quality in 2015 and Perspective}

The strategy for the $K^{+} \rightarrow \pi^{+} v \bar{v}$ selection is to require a one track topology, identified as a pion by the PID system, with no in-time photon candidate. The track has to match a kaon candidate in the beam detectors. The understanding of the acquired data was checked by selecting kaon decays in the fiducial region and background events from the beam activity as shown in figure 3. On the left of the figure it is possible to identify all the main kaon decay modes. On the right the background coming from the beam activity is dominated by $\pi^{+} \rightarrow \mu^{+} v$ decays and scattering of the beam particles in the three station of the GTK. In order to maximize the efficiency of the selection only tracks with a momentum in the range $15-35 \mathrm{GeV} / \mathrm{c}$ are considered for the measurement. To estimate the rejection factor for muons and neutral pions a pure selection based on kinematics for $K^{+} \rightarrow \mu^{+} v$ and $K^{+} \rightarrow \pi^{+} \pi^{0}$ was performed. The muon suppression factor was found to be 

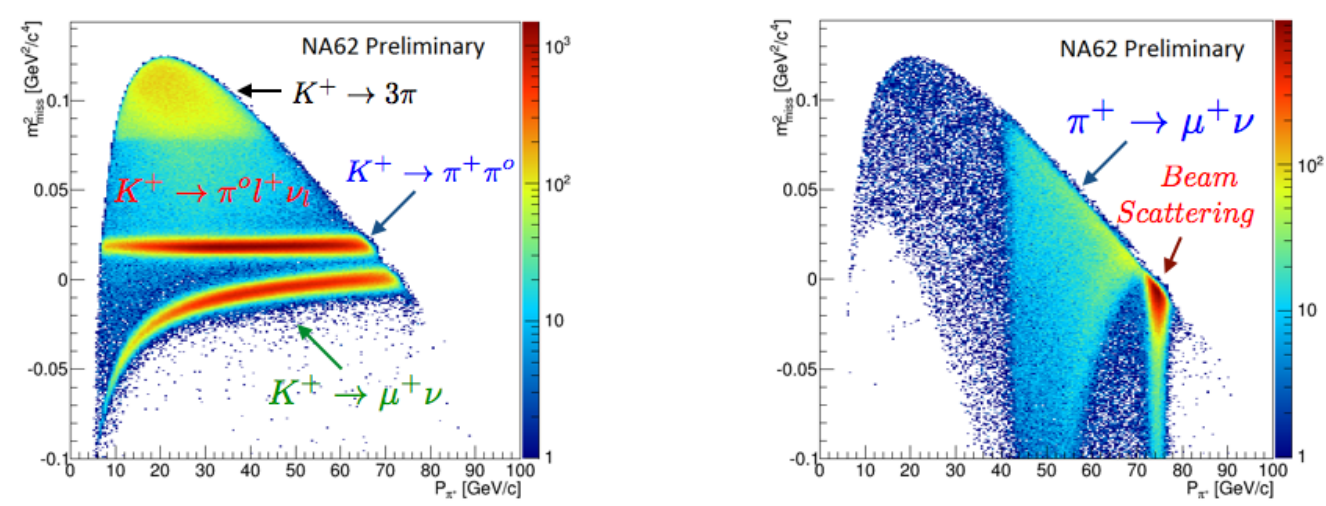

Figure 3: Squared missing mass spectrum as a function of the track momentum. On the left all the main kaon decays requiring a kaon tagging and the vertex in the fiducial region. On the right events, without kaon tagging, from the beam activity: mainly pion decays and scattering of beam particles in the GTK detector.

in line with the expectation for both the RICH, $10^{2}$ with an efficiency of $85 \%$ for pions, and the calorimeters, $10^{4} \div 10^{6}$ with $90 \% \div 40 \%$ efficiency using a simple cut analysis. The $\pi^{o}$ detection inefficiency was estimated to be below $10^{-5}$. The statistics collected in 2015 is the limiting factor to refine the analysis and push the sensitivity towards the needed $\pi^{o}$ rejection power $\left(10^{8}\right)$.

\subsection{Physics Program beyond $K^{+} \rightarrow \pi^{+} v \bar{v}$}

The NA62 apparatus allows to perform a compelling physics program beyond $K^{+} \rightarrow \pi^{+} v \bar{v}$. The main kaon decays can be studied with an unprecedented precision with the opportunity to stress once more the SM predictions. The level of single event sensitivity offers the possibility to further improve the limits on lepton flavour and number violation processes. The $\pi^{o}$ physics can take profit of the efficient photon detection system to search decays in $3 \gamma$ or $4 \gamma$, invisible or dark photon production. The quality of the kinematics reconstruction allows to extend the search for heavy neutrinos in the mass range $100-380 \mathrm{MeV}$ via the $\mathrm{K}^{+} \rightarrow l^{+} v$ decay, improving the existing limits.

\section{References}

[1] V.Fanti et al. (NA48 Collaboration), Nucl. Instrum. Methods A 574, 433 (2007).

[2] C. Lazzeroni et al. (NA62 Collaboration), Phys. Lett. B 719, 326 (2013).

[3] K.A. Olive et al.(Particle Data Group), Chin. Phys. C, 38, 090001 (2014) and 2015 update.

[4] M. Gell-Mann and F. Zachariasen, Phys. Rev. D 124, 953 (1961).

[5] T. Husek and S. Leupold, Eur. Phys. J C 75, 586 (2015).

[6] T. Husek et al., Phys. Rev. D 92, 054027 (2015).

[7] A.J. Buras, D. Buttazzo, J. Girrbach-Noe and R. Knegjens, JHEP 1511, 33 (2005).

[8] S. Adler et al. (E949 and E787 Collaborations), Phys. Rev. D 79, 092004 (2009).

[9] NA62 Technical Design Document, NA62-10-07; https://cdsweb.cern.ch/record/14049857.

[10] G. Ruggiero (NA62 Collaboration), PoS KAON 13032 (2013). 